\title{
Multifunction RF Filters Using OTA
}

\author{
Norbert Herencsar ${ }^{1}$, Kamil Vrba ${ }^{1}$ \\ ${ }^{1}$ Dept. of Telecommunications, \\ Faculty of Electrical Engineering and Communication, \\ Brno University of Technology, Purkynova 118, 61200 Brno, Czech Republic \\ herencsar@phd.feec.vutbr.cz, vrbak@feec.vutbr.cz
}

\begin{abstract}
In this paper the procedure of RF filter design using two active elements OTA is shown. The general method of filter design using OTA amplifiers, when the fully connected feedback network was designed around the two OTA elements, is shown. Autonomous circuits were gradually picked out from this general arrangement, using two to five passive elements. These autonomous circuits then serve as an initial circuit in designing different types of frequency filters. On a selected autonomous circuit, all the procedure of multifunction filter design is shown. The properties of the proposed secondorder multifunction filter were subjected to an AC analysis in the PSpice software [8], a generalized sensitivity analysis and experimental measurement.
\end{abstract}

Keywords: OTA, frequency filter, autonomous circuit, general admittance network, sensitivity analysis.

\section{Introduction}

In the last few years a number of new active elements for designing RF filters working in the current or the voltage mode were developed. Primarily these are CFA (Current Feedback Amplifier), OTA (Operational Transconductance Amplifier), BOTA (Balanced Output OTA), MOTA (Multiple Output OTA), active element working in the mixed mode CDBA (Current Differencing Buffered Amplifier), CC (Current Conveyor), VC (Voltage Conveyor), COA (Current Operational Amplifier) or pure current element CMI (Current Mirror and Inverter). Numerous scientific papers and publications dealing with RF filters using these active elements have been presented [3], [4]. Unfortunately, most of these elements are only on the theoretical level or are currently being developed. Commercially available amplifiers are only OTA and BOTA amplifiers, e.g. MAX43/MAX436 by MAXIM-Dallas Semiconductor [9], LM13600/LM13700 by National Semiconductor or LT1228 by Linear Technology [10].

This paper is focused on the design procedure of RF filters with two transconductance amplifiers, which are referred to as OTA amplifiers. These filters are often appropriate for signal processing of high-speed data communication systems, in cable modems or in hard--drive communication interfaces.

Please use the following format when citing this chapter:

Herencsar, N., Vrba, K., 2007, in IFIP International Federation for Information Processing, Volume 245, Personal

Wireless Communications, eds. Simak, B., Bestak, R., Kozowska, E., (Boston: Springer), pp. 557-568. 


\section{Transconductance amplifiers}

Operational transconductance amplifiers with a single output (OTA) were made commercially available for the first time in 1969 by RCA. The first publications with OTA came out in 1985, when R. L. Geiger and S. E. Sánchez presented to the general public the new CMOS OTA architectures and new circuits of frequency filters [2] with this new active element. The schematic symbol of OTA is shown in Fig. 1a. For tuned RF filters it is appropriate to use an OTA element with variable values of transconductance $g_{\mathrm{m}}$ set by the control current $I_{\mathrm{SET}}$ (Fig. 1b).

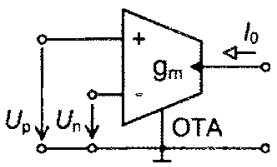

a)

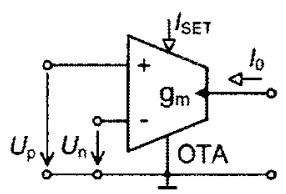

b)

Fig. 1. Schematic symbol of OTA: a) with constant transconductance, b) with variable transconductance.

The ideal OTA is a voltage-controlled current source characterized by transconductance $g_{\mathrm{m}}$. The output current of the OTA element is given by the following equation:

$$
I_{0}=-g_{\mathrm{m}}\left(U_{\mathrm{p}}-U_{\mathrm{n}}\right),
$$

where $U_{\mathrm{p}}$ and $U_{\mathrm{n}}$ are the voltages on non-inverting and inverting inputs of OTA with respect to the ground. The ideal OTA amplifier is characterized by a finite, frequency-independent transconductance $g_{\mathrm{m}}$ while its input and output impedances are theoretically infinite. An important feature of OTAs is the possibility of driving the transconductance $g_{\mathrm{m}}$ by the control current $I_{\mathrm{SET}}$.

Currently, the OTA elements are supplied on the market by many manufacturers. A commercially available OTA element, as mentioned above, is the circuit LT1228 (Linear Technology) [10] or MAX435 (MAXIM-Dallas Semiconductor) [9], which is a high-speed wideband transconductance amplifier (WTA) with high-impedance inputs and output. Due to its unique performance features, it is suitable for a wide variety of applications such as high--speed instrumentation amplifiers, wideband, high-speed RF filters, and high-speed differential line driver and receiver applications. In this paper we deal with the filtering applications of OTA amplifiers. 


\section{General design of autonomous circuits with two OTA elements}

In our workplace, the method of generalized frequency filter design, when the initial circuit is a full-admittance network connected to active elements [5], [6], was developed. The same procedure was used in the case of OTA elements. The basic general autonomous circuit is shown in Fig. 2.

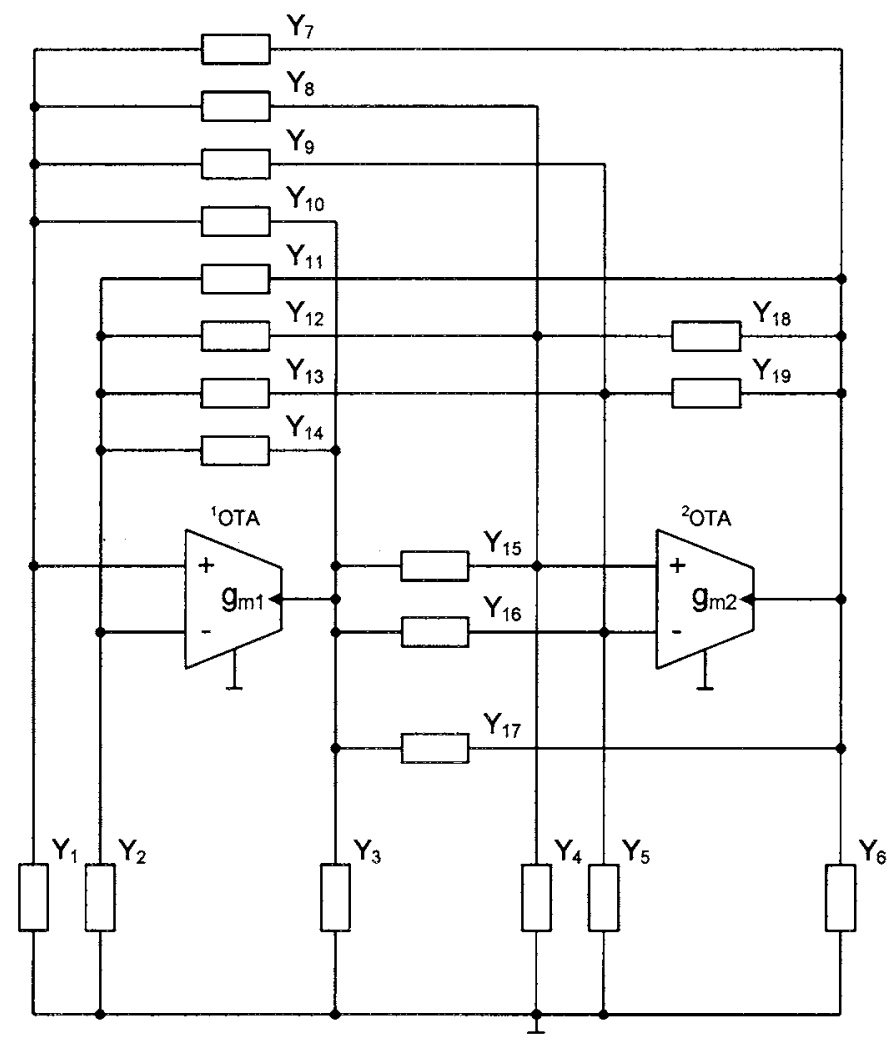

Fig. 2. Full admittance network connected to two OTA elements.

Simpler autonomous circuits were gradually picked out from this general arrangement. We tried to have the passive elements grounded with one terminal, because such elements are easier to implement in integrated circuits. The selected autonomous circuits presented in Table 1 have three or four admittances. In Table 1 the characteristic equations are also given, which describe the behaviour of autonomous circuit. Only their left side is mentioned, the right side is always equal to zero. The characteristic equations were obtained using the SNAP software [7]. 
Table 1. Designed autonomous circuits and their characteristic equations.

No. Autonomous circuits and their left side of characteristic equations




\section{Filter design}

For the verification of the correct function of filters with OTA elements autonomous circuit No. 1, Table 1, has been selected. The characteristic equation of this circuit is:

$$
D=Y_{1} Y_{2}+Y_{2} Y_{3}+g_{m 1} g_{m 2}=0 \text {. }
$$

Due to choosing the passive elements $Y_{1}=G_{1}, Y_{2}=\boldsymbol{p} C_{1}, Y_{3}=\boldsymbol{p} C_{2}$ equation (2) changes to a form which satisfies the feasibility conditions of the frequency filter

$$
D \boldsymbol{p}=\boldsymbol{p}^{2} C_{1} C_{2}+\boldsymbol{p} G_{1} C_{1}+g_{\mathrm{m} 1} g_{\mathrm{m} 2},
$$

where $\boldsymbol{p}=j \omega$ is the complex variable. The frequency filter designed in the voltage mode (VM) is shown in Fig. 3.

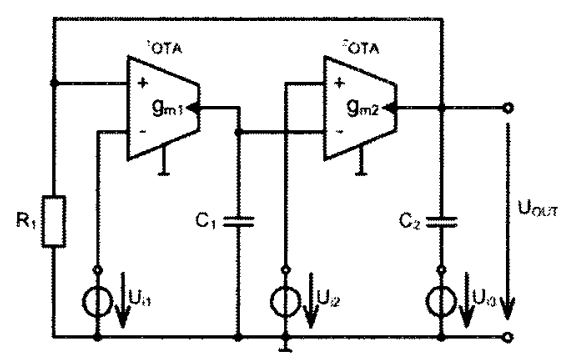

Fig. 3. Designed filter working in VM.

The complex voltage transfer functions of the designed filter with driving voltages $U_{\mathrm{i} 1}, U_{\mathrm{in} 2}$ and $U_{\mathrm{in} 3}$ have the form:

$$
\begin{gathered}
\boldsymbol{K}_{\mathrm{IF}}=\frac{U_{\text {out }}}{U_{\mathrm{i} 1}}=\frac{g_{\mathrm{m} 1} g_{\mathrm{m} 2}}{D \boldsymbol{p}}, \\
\boldsymbol{K}_{\mathrm{BP}}=\frac{U_{\text {out }}}{U_{\mathrm{i} 2}}=-\frac{\boldsymbol{p} C_{1} g_{\mathrm{m} 2},}{D \boldsymbol{p}} \\
\boldsymbol{K}_{\mathrm{HF}}=\frac{U_{\text {aut }}}{U_{\mathrm{i} 3}}=\frac{\boldsymbol{p}^{2} C_{1} C_{2}}{D \boldsymbol{p}}, \\
\boldsymbol{K}_{\mathbf{B R}}=\frac{U_{\text {out }}}{U_{\mathrm{i} 1}+U_{\mathrm{i} 3}}=\frac{\boldsymbol{p}^{2} C_{1} C_{2}+g_{\mathrm{m} 1} g_{\mathrm{m} 2}}{D \boldsymbol{p}}, \\
\boldsymbol{K}_{\mathbf{A F}}=\frac{U_{\text {out }}}{U_{\mathrm{i} 1}+U_{\mathrm{i} 2}+U_{\mathrm{i} 3}}=\frac{\boldsymbol{p}^{2} C_{1} C_{2}-\boldsymbol{p} C_{1} g_{\mathrm{m} 2}+g_{\mathrm{m} 1} g_{\mathrm{m} 2}}{D \boldsymbol{p}}
\end{gathered}
$$

In the voltage mode the circuit according to Fig. 3 can be used as a low-pass (4), band-pass (5), high-pass (6), band-reject (7) or an all-pass (8) filter. 
The characteristic frequency of all filters is:

$$
\omega_{0}=\sqrt{\frac{g_{\mathrm{m} 1} g_{\mathrm{m} 2}}{C_{1} C_{2}}}
$$

The quality factor $Q_{0}$ of all filters is given by the following equation:

$$
Q_{0}=\frac{1}{G_{1}} \sqrt{\frac{C_{2} g_{\mathrm{ml}} g_{\mathrm{m} 2}}{C_{1}}} .
$$

For the required values $Q_{0}$ and $\omega_{0}$, and the selected values $C_{1}$ and $C_{2}$ we can determine other parameters necessary for the design:

$$
g_{\mathrm{m} 1}=\omega_{0} Q_{0} C_{1}, \quad G_{1}=g_{\mathrm{m} 2}=\frac{\omega_{0} C_{2}}{Q_{0}} .
$$

The filter example was designed for the tunable range of characteristic frequency $f_{0} \approx$ $1 \mathrm{MHz}$ to $10 \mathrm{MHz}$, the range of controlling the quality factor $Q_{0}=0.5$ to 5 based on the Butterworth approximation [1]. Capacitors $C_{1}=220 \mathrm{pF}$ and $C_{2}=110 \mathrm{pF}$ were chosen. Other parameters are within these limits: $g_{\mathrm{m} 1,2}=1 \mathrm{~mA} / \mathrm{V}$ to $10 \mathrm{~mA} / \mathrm{V}$, and $R_{1}$ $=100 \Omega$ to $1 \mathrm{k} \Omega$. Commercially available transconductance amplifiers (OTA) are the MAX436 or LT1228 circuits. Their company models were used in the computer simulation in the PSpice software [8]. The simulation results for the voltage-mode second-order multifunction filter are shown in Fig. 4. The characteristics of a filter using LT1228 and MAX436 are compared with the ideal models of OTA amplifiers. From the results it is evident that when using MAX436 the results are close to the ideal curves. The possibility of tuning the characteristic frequency and the quality factor is shown in Fig. 5. For a second-order low-pass filter using LT1228 and MAX436 the two cut-- off frequencies and the mid frequency of tuning $f_{0} \approx 1 \mathrm{MHz}$ to $10 \mathrm{MHz}$ and $Q_{0}=0.5$ to 5 are shown. From the results it is again evident that for filters working at a higher frequency it is preferable to use the MAX436 elements. A specific filter solution is shown in chapter six.

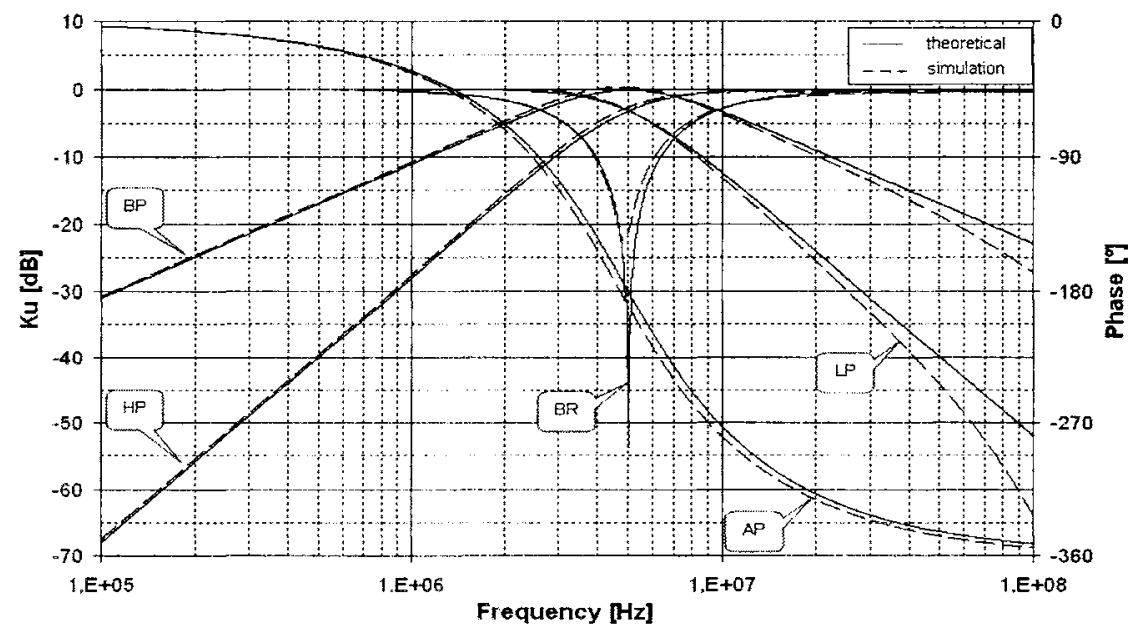




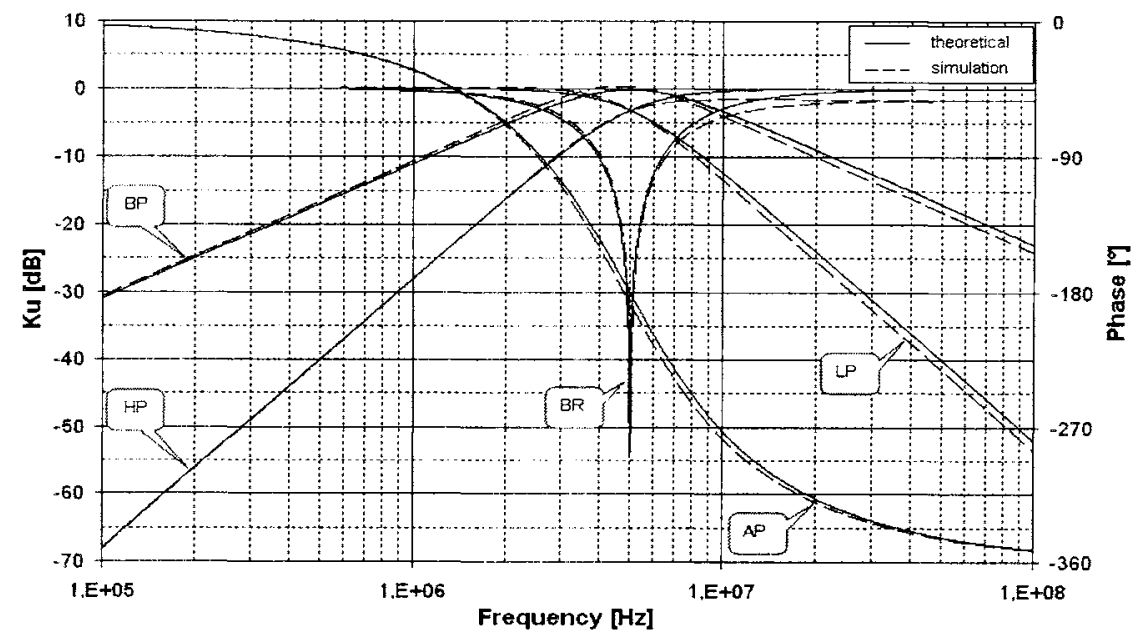

b)

Fig. 4. Frequency characteristics of multifunction filter shown in Fig. 3 using: a) LT1228 and b) MAX436.

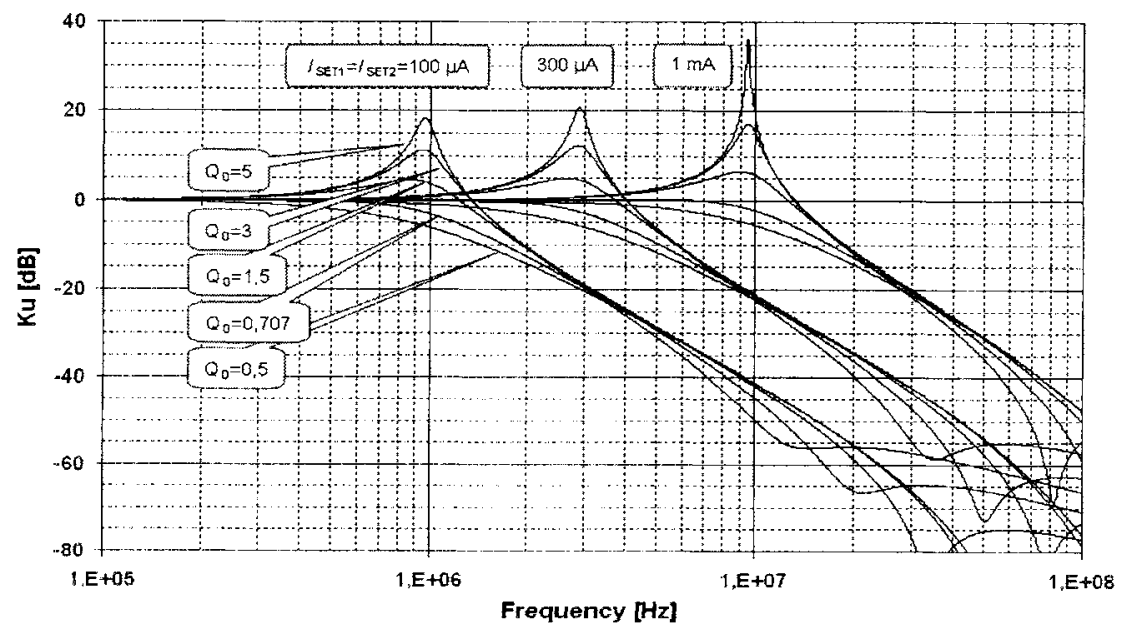

a) 


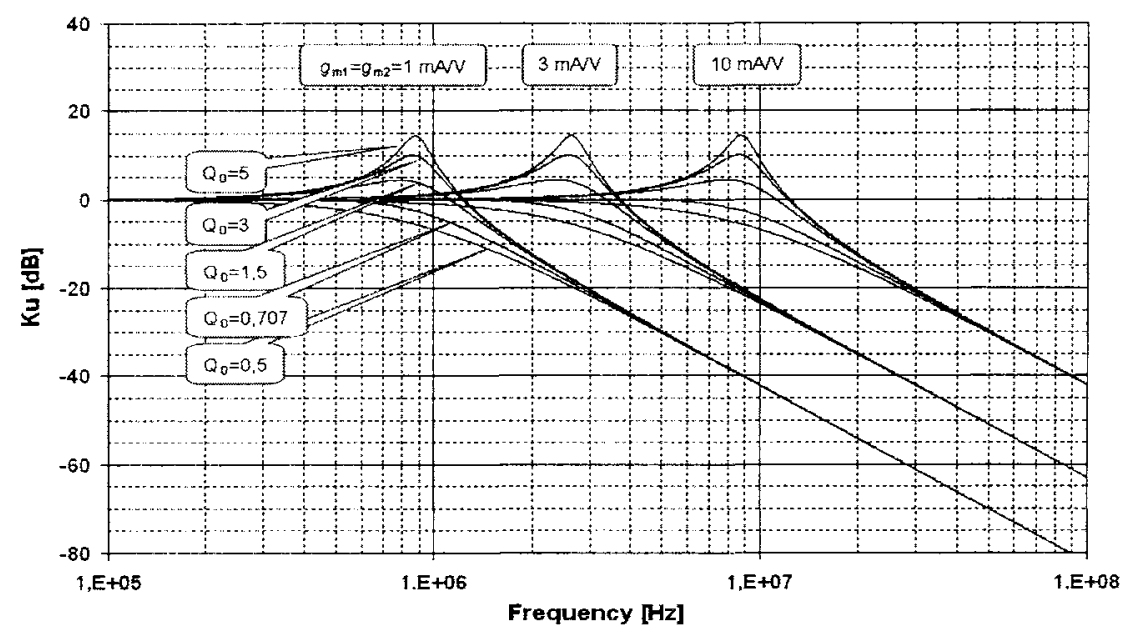

b)

Fig. 5. Frequency characteristics of tuned low-pass filter shown in Fig. 3 using: a) LT1228 and b) MAX436.

\section{Sensitivity function of multifunction filter}

All filters have in their denominator the same left side of characteristic equation (3) of the circuit function and thus the characteristic frequency; the quality factor $Q_{0}$ and the relevant relative sensitivities are identical for all types of filters [3]. For the circuit in Fig. 3 the particular relative sensitivities of characteristic frequency $\omega_{0}$ of all filters are given by the following equation:

$$
-\mathrm{S}_{\mathrm{C}_{1}}^{\omega_{1}}=-\mathrm{S}_{\mathrm{C}_{2}}^{a_{2}}=\mathrm{S}_{\mathrm{g}_{\mathrm{mi}}}^{\omega_{1}}=\mathrm{S}_{\mathrm{g}_{\mathrm{m} 2}}^{a_{1}}=\frac{1}{2}, \quad \mathrm{~S}_{\mathrm{G}_{1}}^{a_{\mathrm{j}}}=0
$$

The relative sensitivities of the quality factor $Q_{0}$ are:

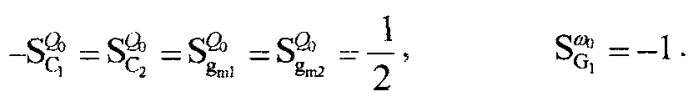

From the results it is evident that the sensitivities of the circuit are low. Potential deviations, which occur in manufacturing or due to changing temperature, can be compensated by changing the values of transconductance $g_{\mathrm{m}}$ by the control current $I_{\mathrm{SET}}$.

In frequency filters the greatest emphasis is put on the overall waveform of the frequency characteristics, and that is why we focus on the effects of parameter tolerances of circuits on overall frequency characteristics. The generalized sensitivity 
function [3] was introduced for this purpose. This function is complex and frequency-dependent, and it gives us overall information about how the transmission will be affected by tolerances of single circuit parameters throughout the frequency band. When we want know how the frequency characteristic is most affected, it is good to define the worst-case relative sensitivity of transmission modulus, which informs about the worst combinations of circuit parameter tolerances. Partial generalized sensitivity functions of filters to active and passive elements are:

$$
\begin{aligned}
& L P S_{G_{1}}^{K_{\mathrm{u}}}=-\frac{\boldsymbol{p} C_{1} G_{1}}{\boldsymbol{p}^{2} C_{1} C_{2}+\boldsymbol{p} C_{1} G_{1}+g_{\mathrm{m} 1} g_{\mathrm{m} 2}}, \\
& { }^{L P} S_{g_{\mathrm{m} 1}}^{K_{\mathrm{u}}}={ }^{L P} S_{g_{\mathrm{m} 2}}^{K_{\mathrm{u}}}=-{ }^{L P} S_{C_{1}}^{K_{\mathrm{u}}}=\frac{\boldsymbol{p}^{2} C_{1} C_{2}+\boldsymbol{p} C_{1} G_{1}}{\boldsymbol{p}^{2} C_{1} C_{2}+\boldsymbol{p} C_{1} G_{1}+g_{\mathrm{m}} g_{\mathrm{m} 2}}, \\
& { }^{L P} S_{C_{2}}^{K_{\mathrm{u}}}=-\frac{\boldsymbol{p}^{2} C_{1} C_{2}}{\boldsymbol{p}^{2} C_{1} C_{2}+\boldsymbol{p} C_{1} G_{1}+g_{\mathrm{m} 1} g_{\mathrm{m} 2}}, \\
& { }^{B P} S_{G_{1}}^{K_{u}}=-\frac{p C_{1} G_{1}}{\boldsymbol{p}^{2} C_{1} C_{2}+\boldsymbol{p} C_{1} G_{1}+g_{\mathrm{m} 1} g_{\mathrm{m} 2}}, \\
& -{ }^{B P} S_{g_{\mathrm{m} 1}}^{K_{\mathrm{u}}}={ }^{B P} S_{C_{1}}^{K_{\mathrm{u}}}=\frac{g_{\mathrm{m} 1} g_{\mathrm{m} 2}}{\boldsymbol{p}^{2} C_{1} C_{2}+\boldsymbol{p} C_{1} G_{1}+g_{\mathrm{m} 1} g_{\mathrm{m} 2}}, \\
& { }^{B P} S_{g_{\mathrm{m} 2}}^{\kappa_{\mathrm{u}}}=\frac{\boldsymbol{p}^{2} C_{1} C_{2}+\boldsymbol{p} C_{1} G_{1}}{\boldsymbol{p}^{2} C_{1} C_{2}+\boldsymbol{p} C_{1} G_{1}+g_{\mathrm{m} 1} g_{\mathrm{m} 2}}, \\
& { }^{B P} S_{C_{2}}^{K_{\mathrm{u}}}=-\frac{\boldsymbol{p}^{2} C_{1} C_{2}}{\boldsymbol{p}^{2} C_{1} C_{2}+\boldsymbol{p} C_{1} G_{1}+g_{\mathrm{m} 1} g_{\mathrm{m} 2}}, \\
& { }^{P} S_{G_{1}}^{K_{\mathrm{u}}}=-\frac{\boldsymbol{p} C_{1} G_{1}}{\boldsymbol{p}^{2} C_{1} C_{2}+\boldsymbol{p} C_{1} G_{1}+g_{\mathrm{m} 1} g_{\mathrm{m} 2}}, \\
& { }^{H P} S_{g_{\mathrm{m} 1}}^{K_{\mathrm{u}}}=H P S_{g_{\mathrm{m} 2}}^{K_{\mathrm{u}}}=-\frac{g_{\mathrm{m} 1} g_{\mathrm{m} 2}}{\boldsymbol{p}^{2} C_{1} C_{2}+\boldsymbol{p} C_{1} G_{1}+g_{\mathrm{m} 1} g_{\mathrm{m} 2}}, \\
& { }^{H P} S_{C_{1}}^{K_{\mathrm{u}}}=\frac{g_{\mathrm{m} 1} g_{\mathrm{m} 2}}{\boldsymbol{p}^{2} C_{1} C_{2}+\boldsymbol{p} C_{1} G_{1}+g_{\mathrm{m} 1} g_{\mathrm{m} 2}}, \\
& { }^{P P} S_{C_{2}}^{K_{\mathrm{u}}}=\frac{\boldsymbol{p} C_{1} G_{1}+g_{\mathrm{m} 1} g_{\mathrm{m} 2}}{\boldsymbol{p}^{2} C_{1} C_{2}+\boldsymbol{p} C_{1} G_{1}+g_{\mathrm{m} 1} g_{\mathrm{m} 2}} .
\end{aligned}
$$

For easier interpretation, the generalized sensitivity functions were expressed graphically. The characteristic frequency $f_{0} \approx 5 \mathrm{MHz}$ and quality factor $Q_{0}=0.707$ based on the Butterworth approximation $\left(b_{1}=1.4142, b_{2}=1\right)$ [1] were chosen. For component parameters the recommendation is: capacitor values $C_{1}=220 \mathrm{pF}, C_{2}=$ $110 \mathrm{pF}$, admittance and transconductance values are $G_{1}=g_{\mathrm{m} 1}=g_{\mathrm{m} 2} \approx 4.9 \mathrm{~mA} / \mathrm{V}$.

The partial generalized sensitivities of transmission are shown in Fig. 3. The relative sensitivities of filter transmission are on the left side, on the right side are semirelative sensitivities of the argument. From the graphs it was found that the sensitivities of low-pass and high-pass filters in the transmission band were zero, which could be of advantage. 

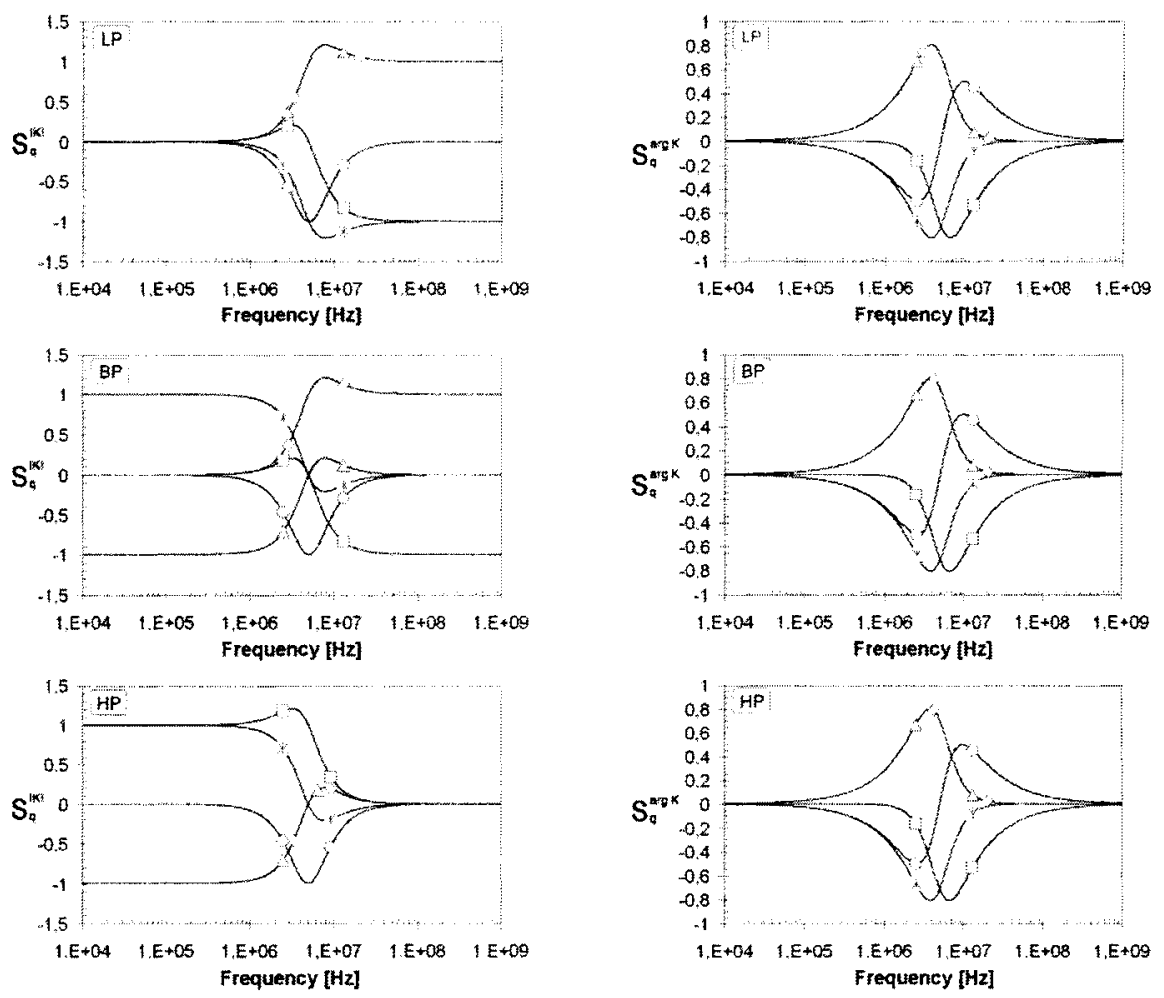

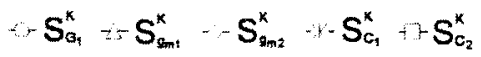

Fig. 6. Partial generalized sensitivities of filter based on circuit in Fig. 3.

The worst-case global relative sensitivity of transmission modulus and the semirelative sensitivity of the filter transmission argument are shown in Fig. 7.
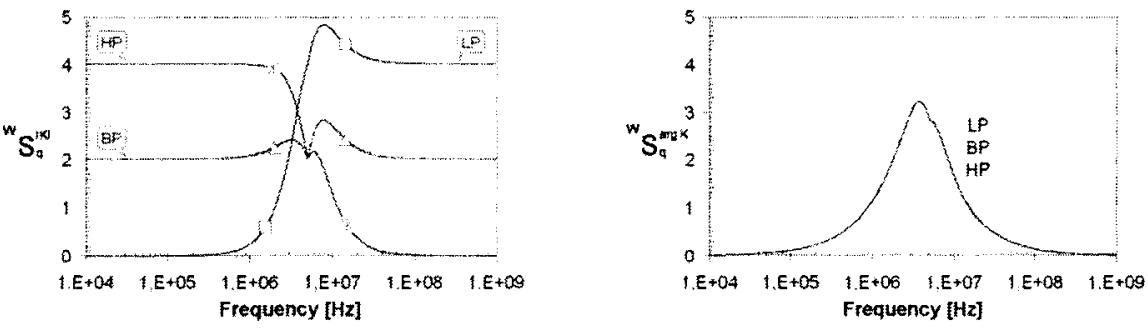

Fig. 7. The worst-case global relative sensitivities of modulus and semi-relative sensitivities of argument for multifunction filter based on circuit in Fig. 3.

The semi-relative sensitivity of transmission argument is the same for all filters. The worst-case sensitivity of the band-pass is approximately identical throughout the frequency band. The sensitivity of high-pass filter in the stop-band is high but in the transmission band it decreases to zero. Similarly, the sensitivity of the low-pass in the stop-band is worse but in the transmission band it is zero. 


\section{Experimental results}

The band-reject filter was chosen for the realization. To set the transconductances of OTA amplifiers the AD5258 [11] digital potentiometers were used. Capacitors $C_{1}=$ $220 \mathrm{pF}$ and $C_{2}=110 \mathrm{pF}$, resistor $R_{1}=200 \Omega$ and transconductances $g_{\mathrm{m} 1,2}=4,9 \mathrm{~mA} / \mathrm{V}$ were used in the realization of the RF filter. A simplified schematic of the system measured is in Fig. 8.

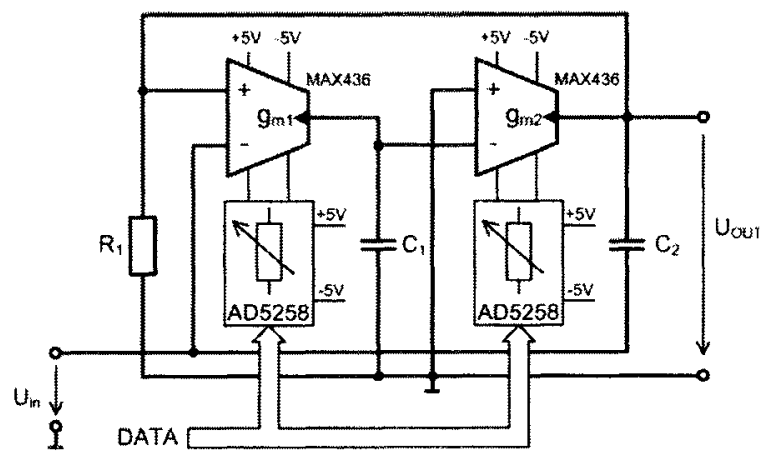

Fig. 8. Schematic of measured band-reject.

The measurement was carried out with an HP3589A network/spectrum analyzer connected to computer via the GPIB bus system. The frequency response measured for the second-order band-reject frequency filter is given in Fig. 9. The results of measurement are matches to simulations.

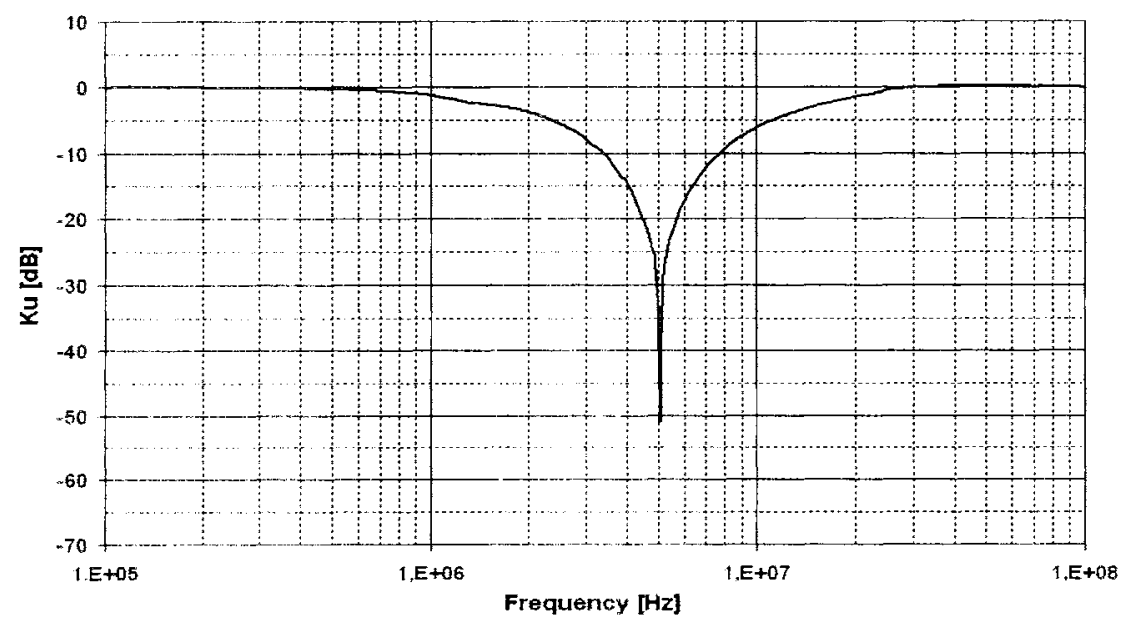

Fig. 9. Frequency response measured for second-order band-reject filter. 


\section{Conclusion}

In this paper a general method for designing new circuits of frequency filters with two OTA elements is presented. Usually, when proposing autonomous circuits we proceed intuitively. The method of connecting a general network of passive elements to two OTAs is shown. Simpler autonomous circuits were gradually eliminated from this general arrangement. An example of the procedure of frequency filter design with two OTA elements is shown. A specific filter solution was simulated in the PSpice software [8], MAX436 [9] and LT1228 [10] company models were used. A generalized sensitivity analysis is shown. The frequency filter designed is expected to be used in the signal processing of high-speed data communication systems, in cable modems or in hard-drive communication interfaces. The band-reject filter was realized experimentally using a MAX436 [9].

Acknowledgment. The paper has been supported by the Czech Science Foundation project GACR 102/06/1383 and Ministry of Education, Youth and Sport of the Czech Republic project No. 1ET301710508.

\section{References}

1. Tietze, U., Schenk, Ch.: Halbleiter - Schaltungtechnik, Springer, 12 ed., 2002. ISBN 3540-42849-6.

2. Geiger, R. L., Sánchez, S. E.: Active Filter Design Using Operational Transconductance Amplifiers: A Tutorial, IEEE Circuits and Devices Magazine, Vol. 1, pp. 20-32, 1985.

3. Chen, W. K.: The Circuits and Filters Handbook. CRC Press, Inc. Boca Raton, Florida, 1995.

4. Deliyannis, T., Sun, Y., Fidler, J. K.: Continuous-Time Active Filter Design, CRC Press, Boca Raton, 1999, 443 pages, ISBN 0-8493-2573-0.

5. Koton, J., Vrba, K.: Method for Designing Frequency Filters using Universal Current Conveyors, International Transactions on Computer Science and Engineering, 2005, Vol.13, No.1, pp. 144-154.

6. Herencsár, N., Vrba, K.: Method for Designing Frequency Filters Using BOTA. In Proceedings of the Second International Conference on Systems, ICONS 2007. SainteLuce, Martinique: IEEE Computer Society, 2007, pp. 1-6. ISBN: 0-7695-2807-4.

7. Biolek, D.s Kolka, Z: SNAP - symbolic, semisymbolic, and numerical analysis of electronic circuits. URL http://snap.webpark.cz/indexa.html

8. OrCAD, Inc. OrCad PSpice User's Guide [pdf online file] USA: OrCAD, 1998. http://www.electronics-lab.com/downloads/schematic/013/. 436 pages.

9. MAX435/MAX436 - Wideband Transconductance Amplifier with Differential Output. Datasheet, MAXIM - Dallas Semiconductor, 1993

10. LT1228 - 100MHz Current Feedback Amplifier with DC Gain Control. Datasheets, Linear Technology, 1994.

11. AD5258 - Nonvolatile, $\mathrm{I}^{2} \mathrm{C}^{(}$-Compatible 64-Position, Digital Potentiometer, Analog Devices, 2007. 